\title{
Sensing some tension
}

Cells are under mechanical tension in their native environment. New genetically encoded tension sensors can make a broader range of these forces visible.

Cells interact with their surrounding extracellular matrix through focal adhesions. The molecules in these structures are under tension, but the mechanisms of tension sensing are not well characterized, in part because of the lack of suitable force sensors in different force ranges.

Carsten Grashoff and his team at the Max Planck Institute for Biochemistry in Martinsried, Germany, have developed two sensors, HP35-TS and HP35st-TS, that extend the range of forces measurable with genetically encoded sensors. Their design is based on the TSMod sensor, which is suitable for measuring forces in the range of 1-6 piconewtons. Each sensor consists of a peptide that unfolds when subjected to tension, and this unfolding is reported by a pair of fluorescent proteins that flank the elastic peptide and serve as a fluorescence resonance energy transfer (FRET) pair. Thus, when the sensor is not under tension, the two fluorescent proteins are close, resulting in increased fluorescent emission. This emission decreases when the two fluorescent proteins are pulled apart.

The force-sensitive portion of HP35-TS stems from the villin headpiece. This sensor detects tension in the range of 6-8 piconewtons, whereas a mutation in the elastic peptide shifts the range of measurable forces to 9-11 piconewtons in HP35st-TS. These developments make forces beyond the previously measurable range accessible.

Grashoff and his team used the new sensors to assess the forces on talins, which link integrin receptors in focal adhesions to the actin cytoskeleton. They observed distinct populations of talins subject to different force ranges, and they found that tension depends on the association of talins with actin and adapter proteins. These different talin populations could be appreciated only because of the availability of force sensors with different sensitivities.

A caveat of force sensors, Grashoff points out, is that the force measurements are performed in bulk. The imaged signal thus represents the force averaged over many molecules, and it is not possible to determine whether all molecules are subject to the same forces or whether certain molecules are under high tension while others are not mechanically engaged. Such questions are interesting and await further sensor developments and possibly the application of superresolution techniques.

Nina Vogt

RESEARCH PAPERS

Austen, K. et al. Extracellular rigidity sensing by talin isoform-specific mechanical linkages. Nat. Cell Biol. 17, 1597-1606 (2015). 